\title{
Does Learning Alternations Affect Phonotactic Judgments?
}

\author{
Presley Pizzo and Joe Pater \\ University of Massachusetts Amherst
}

\section{Background}

It has long been recognized that the phonotactics and alternations of a language share some generalizations in common. For instance, Russian words lack obstruent sequences that disagree in voicing, and also has voicing assimilation alternations to prevent the creation of such sequences (Jakobson, 1978). Chomsky $\&$ Halle (1968) advocated against redundantly representing these generalizations in favor of encoding them as rules that apply both across forms of a morpheme and "internally to a lexical item" (p. 382). Similarly, Kisseberth notes that morpheme structure conditions, that is, phonotactic generalizations, often effect the same result as some of the rules in a language (Kisseberth, 1970:294). In order to capture the insight that multiple generalizations are driven by one force, we must assume that phonotactics and alternations are encoded in the same grammar.Optimality Theory (Prince \& Smolensky, 1993/2004) formalizes this insight by using one set of constraints to model both phonotactics and alternations.

However, there are also cases where phonotactics and alternations do not work towards the same end results, but instead alternations avoid surface forms that are phonotactically acceptable. For instance, Pierrehumbert (2006) shows that the velar softening rule in English generalizes to novel Latinate-sounding words, such as $[\mathrm{kl} \varepsilon \mathrm{mik}] \sim[\mathrm{kl}$ misisiri]. In addition to her arguments that velar softening is not phonetically grounded, she shows that it does not generalize to Germanic-sounding words such as [blek] $\sim$ [blekiri], indicating that the alternations in the Latinate words is not necessarily required by the phonotactics.

Pater \& Tessier (2003) investigated the relationship between phonotactics and alternations in adults, by asking whether knowledge of a phonotactic generalization affected the ease with which an alternation is learned. If the two kinds of knowledge are held in the same system, then an alternation motivated by a known phonotactic generalization should be easier to learn than one motivated by a previously unsupported phonotactic generalization. They found that this was the case, offering support for the view that phonotactics and alternations are either encoded in the same grammar or have a pathway for sharing information. They note, however, that another interpretation of their results is possible: the alternation not supported by English phonotactics is not only novel to English, but phonetically unmotivated. If speakers are biased towards learning phonetically grounded generalizations (see for instance Becker et al. (2011), c.f. Hayes et al. (2009)), this alternation may have been harder to learn for that reason rather than because it lacked support from the phonotactic system.

Even if their result is due to communication between the systems encoding phonotactics and alternations, their experiment investigated only one direction of communication: from phonotactics to alternations. Thus, the question of whether alternation-based knowledge affects phonotactics remains open.

This question is relevant to the way work in phonotactic modeling is carried out. Often, phonotactic models are built to ignore morphemic and lexical information, learning phonotactic generalizations in the absence of alternations (Coleman \& Pierrehumbert, 1997; Bailey \& Hahn, 2001; Vitevitch \& Luce, 2004; Albright, 2009). This has also been true of some constraint-based models in the Optimality Theory tradition. For instance, Hayes \& Wilson (2008) model the induction of phonotactic constraints from words, but these words are not associated with meanings or broken into morphemes, so alternations are not represented. As this learner is used to model adult data, and has been used to assess the learnability of phonotactic generalizations given the lexicon of a language (Daland et al., 2011), it is important to know whether its omission of the influence of alternations on constraint identities and weights is an accurate representation of reality or a simplification.

There are also constraint-based models of learning in the Optimality Theory family that assume that 
alternations and phonotactics do interact (Tesar \& Prince, 2004; Jarosz, 2006). In this case, it is also important to know whether the flow of information the models depend on is actually possible and should be appealed to in order to explain empirical findings.

\section{Experiment 1}

In order to address this gap in our understanding of the interaction of parts of the phonological grammar, we conducted an experiment to test whether learning an alternation affected participants' phonotactic judgments on underived words. It is difficult to find a case in natural language with the properties necessary to do a well-controlled test of this nature, because the phonotactic evidence for the generalization must be controlled in order to test the effect of the alternation. Thus, we use an artificial language learning paradigm.

2.1 Method In order to test whether alternations affect phonotactics, this experiment manipulated alternation-based evidence while keeping phonotactic evidence constant.

Two constraints were constructed: one against a voiced obstruent followed by a voiceless obstruent (specifically [f]), referred to as $* \mathrm{DF}$, and one against a nasal followed by an obstruent of a different place (also [f]), referred to as *MF. One rule was constructed to repair each constraint: disagreement in voicing is repaired by devoicing the first obstruent (Devoicing), and place disagreement is repaired by changing the place of the nasal (Place Assimilation).

Voicing assimilation and place assimilation are differently supported by the English lexicon and the productive processes of English (Kiparsky, 1985; Jun, 2004; Darcy et al., 2009). However, the design of the experiment does not require them to be equally learnable, as the experiment is designed to test the interaction between the rule participants are trained on and the constraint they are tested on, rather than a main effect of the rule.

These constraints and rules guided the construction of words in an artificial language. The language has a plural suffix -[fa], and singular nouns have no suffix. When pluralization is applied to stems ending in voiced obstruents, which in this language include only [b] and [d], *DF is violated and Devoicing applies. When pluralization is applied to stems ending in non-labial nasals, which in this language include [n] and [n], *NF is violated and Place Assimilation applies.

The experiment has a between-participants design, where the participants are divided into two groups and each exposed to a different exposure and training phase. The test phase is the same across these two treatments.

Neither treatment sees any violations of either constraint. However, each treatment only sees direct evidence for one rule. Thus, for each treatment there is an active rule and a hidden rule. For a given treatment, participants are shown both the singular and plural form of stems that undergo the active rule, but only a singular or a plural for each stem that would undergo the hidden rule. Thus, the application of the hidden rule is neither confirmed nor denied. There is phonotactic evidence for the constraint that motivates the hidden rule, because of the lack of violations of it throughout the language, but there is no alternation-based evidence for the hidden rule.

The test phase then poses two-alternative forced choice questions concerning both constraints. For each constraint, there are questions pitting an apparently stem-internal violation of the constraint against a word that satisfies the constraint. Specifically, the constraint-satisfying word is identical to the constraint-violating word except for the first segment of the constraint violation - it is as if the rule has applied to repair the violation.

The dependent variable measured in this experiment is the probability of choosing a constraint-violating word in the test phase. If alternation-based evidence can affect one's phonotactic grammar, there should be an interaction between the treatment a participant is given and the constraint being tested, so that when participants are trained to apply a certain rule, they disprefer violations of the constraint that motivates that rule more than participants who were not trained to apply that rule.

2.1.1 Participants One hundred participants were recruited from Mechanical Turk and paid for their participation. They were all located in the United States and claimed to be over 18 years old and native speakers of English. As in Experiment 1, participants were only run during the hours of noon and 5pm Eastern time on weekdays. Participants were paid $\$ 2.25$, as the experiment was predicted to take up to 15 
minutes.

Participants were excluded from the analysis based on native speaker status, whether they seemed to be paying attention, and whether they seemed to have learned the rules in the training session.

Native speaker status was assessed as in Experiment 1, based on questions about their native language and the language they speak at home. No participants were excluded on this basis, as all responded that they are native and regular speakers of English.

Participants were considered inattentive if they answered too quickly or chose the option on one side of the screen too consistently. Reaction times under $50 \mathrm{~ms}$ are likely due to bots, so any participants with such short times were excluded. Participants who chose the option on the left or the option on the right more than $90 \%$ of the time were also excluded. These criteria were applied and one participant was excluded based on answer speed.

In place of catch trials, we further assessed attention and success at the task through participants' performance in the training phase. The training phase repeated a maximum of five times. If they did not correctly answer $80 \%$ of the graded questions in a training round before getting to the fifth round, they were assumed to have not learned to apply the rule their language supports, and were excluded from the main analysis. Thirty-six participants were excluded on this basis.

In total, 63 participants were included in the analysis.

2.1.2 Inventory The words of the artificial language used in this experiment were presented orthographically. The inventory of the language consisted of the following letters:

(1) Inventory
a. $\quad$ Trigger for both constraints: $f$
b. $\quad$ Triggers for *DF: $b, d$
c. $\quad$ Repairs for *DF: $p, t$
d. $\quad$ Triggers for $* \mathrm{NF}: n, n g^{1}$
e. $\quad$ Repair for *NF: $m$
f. Others: $l, s, a, e, i, o, u$

2.1.3 Exposure Phase The first phase of the experiment after instructions were given was the exposure phase. The purpose of this phase was to begin teaching the participants the phonotactic patterns and the active rule without yet testing their memory. The task in this phase was to simply type the word or words of the artificial language that were displayed on the screen.

This phase consisted of three kinds of items: singular only, plural only, and singular-plural. The singularonly items showed a singular noun from the artificial language. There were ten of these items, and their words all ended in triggers for the hidden rule.

The plural-only items, of which there were also ten, showed a plural noun that ended in a repair for the hidden rule. However, the stems used in singular-only items were never used in plural-only items. Thus, the evidence was consistent with the use of the hidden rule, but did not prove its application.

The singular-plural items, on the other hand, showed a singular noun and the plural version of that same noun. There were fifteen of these: ten showing the active rule, and five showing non-alternating stems. The items showing the active rule had a stem ending in a segment that triggers the active rule, and its plural form, showing that the active rule had applied. For instance, if the active rule was Devoicing, the singular would end in $b$ or $d$, and the stem of the plural would end in $p$ or $t$, respectively.

The items showing the non-alternating words had stems ending in $p, t, m, l$, or $s$. Their plurals violated no constraints and thus consisted of the faithful stem and the suffix $-f a$.

(2) Exposure stimuli examples when Devoicing is the active rule

a. Singular-only (10): lobon

b. Plural-only (10): funemfa

c. Singular-plural, faithful (5): teldus - teldusfa

d. Singular-plural, alternating (10): nemab - nemapfa

\footnotetext{
1 Participants were instructed that in this language, $n g$ is always pronounced as in singer, never as in finger.
} 
(3) Exposure stimuli examples when Place Assimilation is the active rule

a. Singular-only (10): nemab

b. Plural-only (10): funepfa

c. Singular-plural, faithful (5): teldus - teldusfa

d. Singular-plural, alternating (10): lobon - lobomfa

The exposure stimuli were generated using a $\mathrm{CV}(\mathrm{C}) \mathrm{CVC}$ template. The consonants of the inventory were evenly distributed over the stimuli in the first consonantal slot and also in the second mandatory consonantal slot, with the exception that word-initial $n g$ was swapped with the consonant that had been placed in that word's second onset, to avoid distracting participants with an ungrammatical word-initial $n g$. The optional medial consonant was placed in two of the singular-plural faithful stimuli and three of the singular-plural alternating stimuli (for each kind of alternation), in order to show participants that word-internal consonant clusters are allowed in this language, since they appear in the test words. They always consist of consonants that are not triggers for a specific rule, so that their presence doesn't influence answers to the test questions. The final consonant is dictated by the type of stimulus, as it is the one that may undergo rule application. Both vowel slots were filled by evenly distributing the vowels of the inventory over words and positions. The stimuli used in other portions of the experiment were generated similarly: positions whose identities were not dictated by the needs of the experimental design were filled via uniform distribution of sounds from the inventory, except that $n g$ was kept out of word-initial position.

The instructions at the beginning of the exposure block were as follows:

In this part of the experiment, you will learn words from a made-up language.

Sometimes you'll see one word, and sometimes you'll see two: the singular version of the word first, and then the plural version.

To help yourself catch on to this new language, type the words you see into the text box. We recommend pronouncing them out loud, too. In this language, "ng" is always pronounced as in "singer", never as in "finger".

Each exposure question had the following text: "Please write this word in the text box and pronounce it to yourself:" followed by one word or an appropriately pluralized version of that text followed by a singularplural pair of words in the format "lobon - lobomfa". Below was a text box. No pictures or meanings were given to indicate the number of the nonce words, but as participants saw singular-plural pairs in their predictable order, they may have been able to recognize the plural suffix elsewhere.

The materials were generated and then read into a Python script that used Speriment to generate a website that was then launched on Mechanical Turk using PsiTurk.

2.1.4 Training Phase After the exposure phase, participants went through a training phase. The goal of this phase was to ensure that participants had learned the active rule. The task was to choose the correct of two plural forms for a given singular form.

The phase consisted of thirty items: ten for the active rule, ten for the hidden rule, and ten for nonalternating fillers.

The instructions for this phase were as follows:

Now we'll focus on learning singulars and plurals a bit more. You'll see the singular version of a word and two possible plurals. Choose the one you think is correct for this language. Sometimes you'll then be given the correct answer afterwards, to help you learn. Other times you won't see how you did.

The length of this phase depends on your accuracy. We've found that people who take their time on these questions actually finish this phase much faster than those who rush through the questions. Take your time and pronounce the correct answers out loud when they're given to help you learn how this language sounds.

The active rule items showed a singular noun ending in a trigger for the active rule, and presented two potential plural forms: one applying the active rule and one failing to apply it. The participant's response was considered correct if he or she chose the form which applied the rule. Correct responses were followed by a 
page saying "Correct!" and showing the correct singular-plural pair. Incorrect responses were followed by a page saying "No, the correct pairing is" followed by the correct singular-plural pair.

The filler items showed a singular noun ending in $l$ or $s$ and presented a plural form with a faithful stem and a plural form where the final consonant of the stem had been changed from $l$ to $s$ or vice versa. The faithful choice was considered correct. The responses were followed by the same kind of feedback as described for the active rule items.

The hidden rule items showed a singular noun ending in a trigger for the hidden rule and two options for its plural, one with a faithful stem and one having undergone the hidden rule. The participants are not taught whether the hidden rule applies or not, so no feedback was given for these items and they were not considered correct or incorrect.

The participant's score on the active rule and filler items was calculated by Speriment ${ }^{2}$. If the participant had answered less than $80 \%$ of the graded questions correctly, the training block would repeat, with the question order newly shuffled. This would continue until either the participant passed the $80 \%$ mark or the block ran five times, at which point the participant would continue on to the testing phase.

2.1.5 Testing Phase The testing phase was intended to test phonotactic judgments of both constraints. It was identical for participants in both treatments. The task was to choose which of two words sounded more like it belonged in the artificial language. The two words were minimal pairs. Neither contained a plural suffix; rather, both had an $f$ in the middle of the word. The words differed in the segment preceding the $f$. In fillers, they differed randomly. In test items, one of the options would have a segment that triggered a given constraint and the other option would have a segment that repaired that constraint.

(4) Example options on a test item for *DF (20)
a. madfas
b. matfas

(5) Example options on a test item for *NF (20)

a. mangfas

b. mamfas

Example options on a filler item (10)

a. sulfen

b. susfen

As you can see in the examples above, the same word frames were used to create test items for both constraints, while different ones were used for fillers. For each constraint, there are two triggering segments. Each triggering segment was used in half of the word frames for a given rule. Within the half that used one triggering segment for a given rule, half used one triggering segment for the other rule and half used the second triggering segment for the other rule.

2.2 Results The hypothesis that alternations affects phonotactics predicts an interaction between training condition and testing condition. Specifically, if learning a rule makes people disprefer the kind of violation that is removed by that rule, then we predict that participants will prefer violations of the constraint motivating the hidden rule more than they prefer violations of the constraint motivating the active rule, regardless of the rule that was active. The interaction plot in Figure 1 shows strong evidence of such an interaction. Recall that *DF motivates Devoicing and *NF motivates Place Assimilation. When training and testing "match," that is, a participant is trained on a rule and tested on the constraint that motivates that rule, they disprefer violations more than when training and testing do not match. The same means are given, along with standard deviations, in (7).

(7) Percent of times a constraint violation was chosen by condition in Experiment 1

2 https://github.com/presleyp/Speriment 


\begin{tabular}{llll}
\hline Rule Trained On & Constraint Tested On & Mean & Standard Deviation \\
\hline Devoicing & *DF & 51 & 50 \\
Devoicing & *NF & 58.2 & 49.4 \\
Place Assimilation & *DF & 63.3 & 48.2 \\
Place Assimilation & *NF & 40.9 & 49.2 \\
\hline
\end{tabular}

The baseline preference for violations of *DF is higher than the baseline preference for violations of *NF, which is expected given the English place assimilation rule. This offers some reassurance that the task worked as expected. The point of interest in this plot is that the slopes of the lines are opposite, showing that the type of violation participants preferred more depended on the type of training they received, and thus supporting the hypothesis that training, that is, knowledge of alternations, can affect phonotactic judgements.

\section{Interaction of Rule and Constraint}

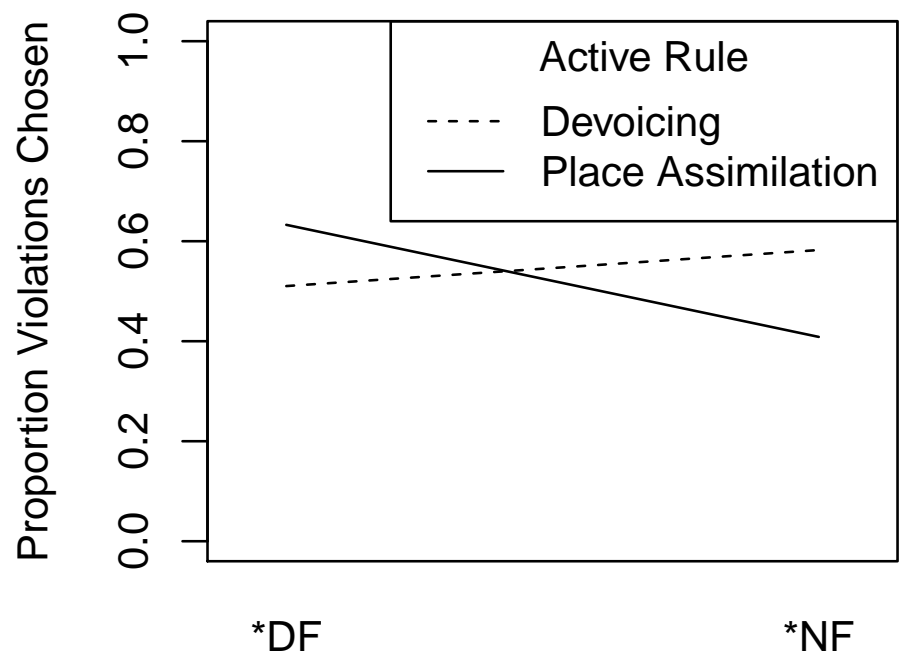

\section{Constraint Tested}

Figure 1: Interaction between effect of rule training and constraint testing in Experiment 1.

For a more detailed view of the results, Figures 2 and 3 show violin plots of data. The former shows a violin plot of the percent of times each participant chose a constraint violation in each condition. Figure 3 shows the same violin plot aggregated by item rather than by participant. As in the interaction plot, the violin plots show that a match between training and testing generally produces lower preferences for violations than a mismatch does. In these figures, Devoicing is abbreviated 'D' and Place Assimilation is abbreviated 'PA.'

Figure 2 shows that the effect of training on Devoicing is subtle; the first two violins are fairly similar looking, although the median and interquartile range show a higher preference for violating *NF than *DF, as predicted. The effect of Place Assimilation training is more obvious. The distribution of participant means for those trained on Place Assimilation when they were tested on *DF is differently shaped than the other three distributions. This shows that no participants chose *DF-violating forms at a low rate after being trained on Place Assimilation.

Figure 3 once again reflects the predicted interaction. As in the grand means and the by-participant data, the effect of Devoicing training was smaller than the effect of Place Assimilation training.

A logistic mixed effects model was fitted to the data using the lme4 package (Bates \& Maechler, 2009) in R (R Development Core Team, 2011). It included random slopes and intercepts for participants and items. 


\section{Preference for Violation by Participant}

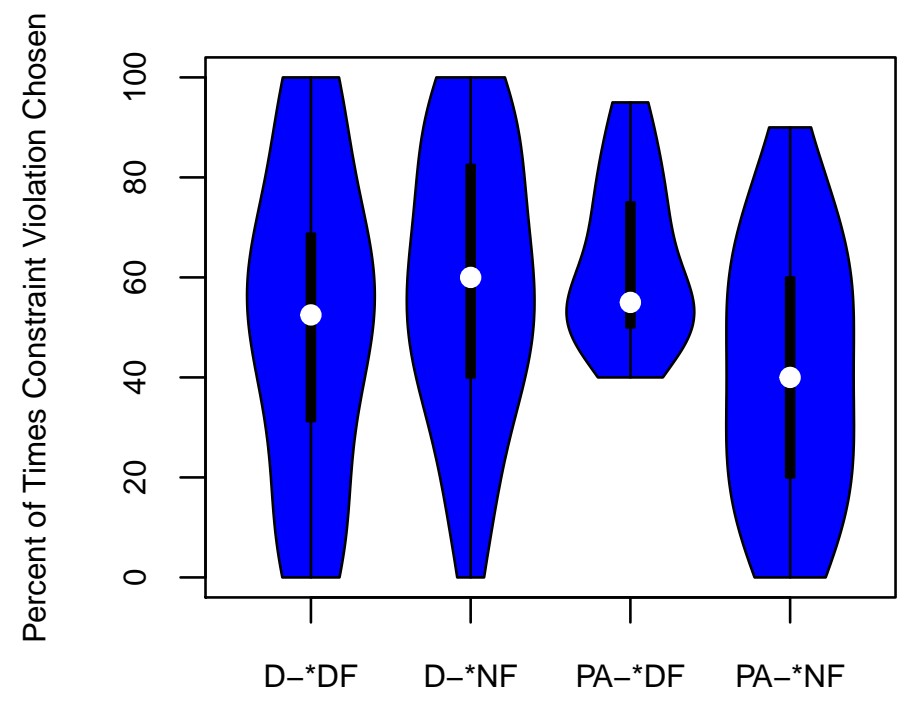

Rule Trained On - Constraint Tested On

Figure 2: Distribution of by-participant violation preference for each condition in Experiment 1.

\section{Preference for Violation by Item}

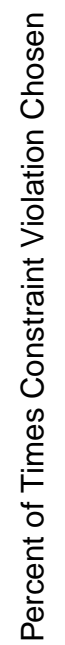

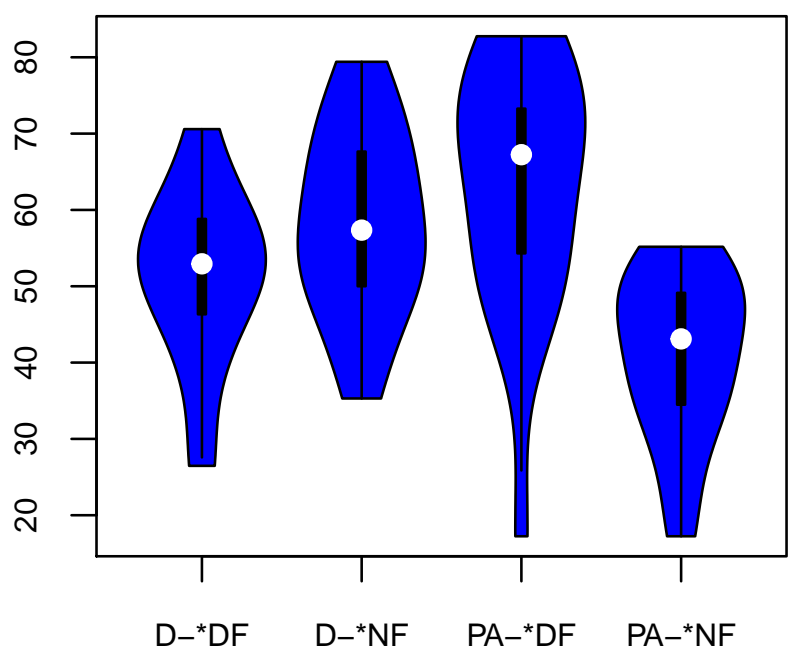

Rule Trained On - Constraint Tested On

Figure 3: Distribution of by-item violation preference for each item in Experiment 1. 
The fixed effects were the training condition, the testing condition, their interaction, and the side of the page the constraint-violating word was presented on. The complete formula is given in (8). The table in (9) shows the coefficients found for this model. It shows that the main effects of training condition and testing condition were not significant. However, as predicted, their interaction was significant, at the $\alpha=0.001$ level. Another significant effect was that of the positioning of the options. Participants were more likely to choose the constraint-violating option if it appeared on the left.

$$
\begin{aligned}
& \text { Mixed effects model formula } \\
& \text { ChoseViolation } \sim \text { Permutation } * \text { Violates }+ \text { ViolationPosition }+(1 \mid \text { Participant })+(0+\text { Permutation } \mid \\
& \text { Participant })+(0+\text { Violates } \mid \text { Participant })+(0+\text { Permutation:Violates } \mid \text { Participant })+(1 \mid \text { Item })+(0 \\
& + \text { Permutation } \mid \text { Item })+(0+\text { Violates } \mid \text { Item })+(0+\text { Permutation:Violates } \mid \text { Item })
\end{aligned}
$$

\begin{tabular}{lll}
\multicolumn{3}{l}{ Coefficients of mixed effects model for Experiment 1} \\
\hline Factor & Estimate & $p$-value \\
\hline Intercept & 0.17 & 0.2906537 \\
Active Rule & -0.1 & 0.4911305 \\
Constraint Tested & -0.2 & 0.2004161 \\
Active Rule:Constraint Tested & -0.42 & 0.0018844 \\
Violation Position & -0.42 & $2.055024 \times 10^{-5}$ \\
\hline
\end{tabular}

2.3 Discussion The results support the hypothesis that knowledge gleaned from alternations can affect the phonotactic grammar, and motivate research on phonotactics that takes the presence of alternations into account. The modeling of phonotactics alone may still be useful as a methodological abstraction, but this study suggests that it will be important to consider how this simplification may skew the results. This study also bears on work on derived environment effects. Such effects, reviewed in Wolf (2008), occur when a rule applies at a morpheme boundary but not in a monomorphemic context. By showing the generalization of a rule learned at a morpheme boundary to a presumably monomorphemic context, these results suggest that the presence of derived environment effects may not be the baseline hypothesis of the language learner.

\section{Experiment 2}

The primary weakness of Experiment 1 is that the presence of feedback creates a confound. If participants update their phonotactic grammars of the artificial language based not just on the presence of the test words but also on the presence of feedback for the test words, it would cause participants to prefer forms that satisfy the constraint that motivates their active rule. It is unclear if this effect is plausible, because token frequency, which is increased by feedback while type frequency is held constant, has not been found to be predictive of phonotactic judgments (Albright, 2008). However, feedback may simply amplify attention. In order to address this confound, a second experiment was performed. Experiment 2 removes feedback from the design, resulting in an experiment with an exposure phase, one iteration of training where no feedback is given, and a testing phase.

3.1 Method Experiment 2 is very similar to Experiment 1; the parts of the method that were different are described below.

3.1.1 Training Phase Recall that the training phase in Experiment 1 consisted of questions where a singular form was presented and the participant was asked to choose between two possible plural forms, and then, for some trials, given feedback naming the correct singular-plural pair. The training phase in Experiment 2 used the same stimuli and question format, but eliminated all feedback. Because the lack of feedback made it unlikely that repeated iterations would improve performance, only one iteration was given, regardless of accuracy rate. Due to the lack of feedback, this phase was not truly a training phase, and is called that only for comparison with Experiment 1. Rather, this phase was used to determine which participants had learned the rule sufficiently that they should be included in the analysis. It is possible, though, that participants organized and solidified their knowledge of the rule by answering the questions in this phase. 
3.1.2 Testing Phase The testing phase was identical except that, in order to increase the validity of the assumption that participants were treating test words as underived, the instructions to the test phase specified that unlike the words seen previously, these did not have any suffixes added.

3.1.3 Participants Participants were once again run on Mechanical Turk. Because the experiment was shorter, they were paid $\$ 1.10$ each, under the assumption that the experiment would take about 7 minutes to complete. All exclusion criteria from Experiment 1 were used except the number of iterations, which was not applicable because multiple iterations were not possible in Experiment 2. These criteria resulted in two participants whose native languages were not English being excluded from the analysis.

In place of number of iterations as a metric of whether participants learned the alternation, percent correct answers in the single training iteration was used. In Experiment 1, 46 of 99 participants reached criterion in their first iteration of training. In order to get a similar amount of usable data, 200 participants were run on Experiment 4. However, due to the lack of feedback, which could help participants learn even within one iteration, a lower percentage of participants reached the original criterion of $80 \%$ correct, yielding 57 participants. To increase the likelihood of having sufficient data, the criterion was lowered to $70 \%$ accuracy. This decision was made before analyzing any data to avoid anti-conservative effects due to data peeking. This resulted in 80 participants being included in the analysis.

\subsection{Results}

(10) Percent of times a constraint violation was chosen by condition in Experiment 2

\begin{tabular}{llll}
\hline Rule Trained On & Constraint Tested On & Mean & Standard Deviation \\
\hline Devoicing & *DF & 52.1 & 50 \\
Devoicing & *NF & 58.6 & 49.3 \\
Place Assimilation & *DF & 55.7 & 49.7 \\
Place Assimilation & *NF & 49.4 & 50 \\
\hline
\end{tabular}

Figure 4 shows the interaction plot for Experiment 2. Once again, we see that the slopes of the lines have different signs, indicating that training on a rule decreased preferences for violating the constraint that motivates that rule. Compared to Experiment 1, participants trained on Place Assimilation behave less differently on the two constraints.

Figure 5 shows a violin plot of the percent of times each participant chose a constraint violation in each condition. It shows a clear trend towards an effect of training in the Devoicing condition; the preference for violations is lower when the constraint tested is *DF, motivated by the active rule, than when it is *NF. This trend is less clear in the Place Assimilation condition.

Figure 6 shows the same violin plot aggregated by item rather than by participant. In this plot, the trend is clearer in the Place Assimilation condition. As in Experiment 1, items testing *DF among participants trained in Devoicing have a long lower tail, showing that a few items are preferred without the constraint violation. In the Devoicing condition, the trend is more subtle, but still leans in the predicted direction.

The same model structure that was used to analyze the data from Experiment 1 was used to analyze this data, although some random effects were omitted to allow the model to converge. The resulting formula is given in (11). The coefficients found by the model are shown in (12).

\begin{tabular}{|c|c|c|}
\hline $\begin{array}{l}\text { Mixed effects model formula } \\
\text { ChoseViolation } \sim \text { Permutation } \\
\mid \text { Participant })+(0+\text { Violates } \mid \mathrm{P}\end{array}$ & $\begin{array}{l}\text { Violates } \\
\text { ticipant) }\end{array}$ & $\begin{array}{l}\text { iolationPosition }+(1 \mid \\
1 \mid \text { Item })+(0+\text { Perm }\end{array}$ \\
\hline Coefficients of mixed effects mo & el for $\operatorname{Exp}$ & riment 2 \\
\hline Factor & Estimate & $p$-value \\
\hline Intercept & 0.2 & 0.1574127 \\
\hline Active Rule & -0.08 & 0.4456613 \\
\hline Constraint Tested & -0.01 & 0.9693086 \\
\hline Active Rule:Constraint Tested & -0.16 & 0.1040932 \\
\hline Violation Position & -0.34 & $4.1330069 \times 10^{-5}$ \\
\hline
\end{tabular}

As before, there is a significant effect of the position of the constraint-violating option on the page, and 


\section{Interaction of Rule and Constraint}

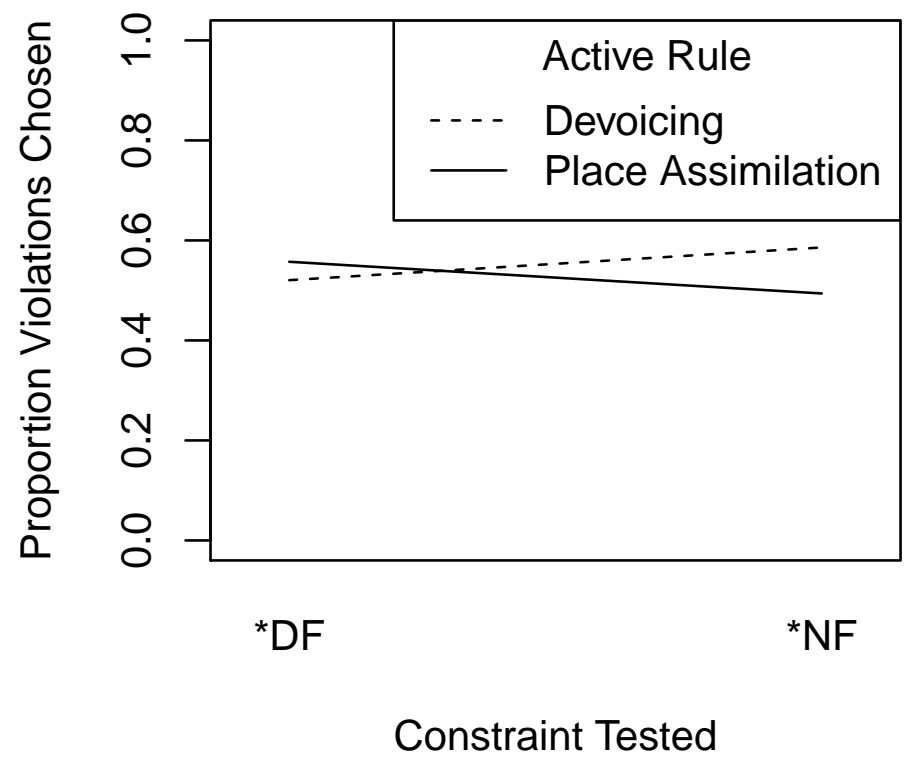

Figure 4: Interaction between rule training and constraint testing in Experiment 2.

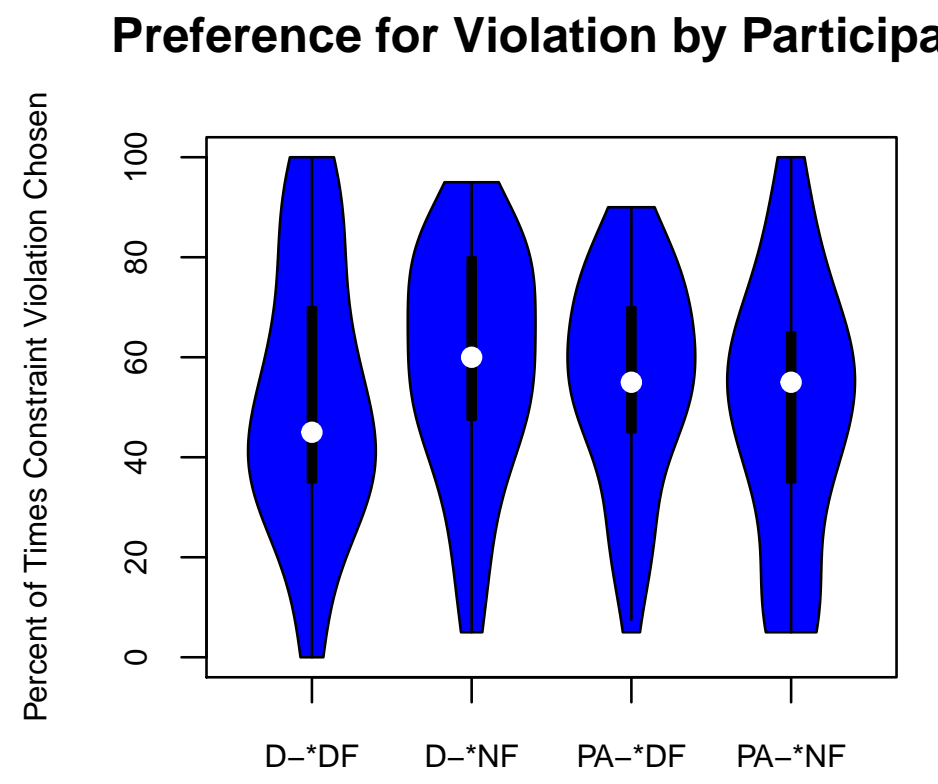

Rule Trained On - Constraint Tested On

Figure 5: Distribution of by-participant violation preference for each condition in Experiment 2. 


\section{Preference for Violation by Item}

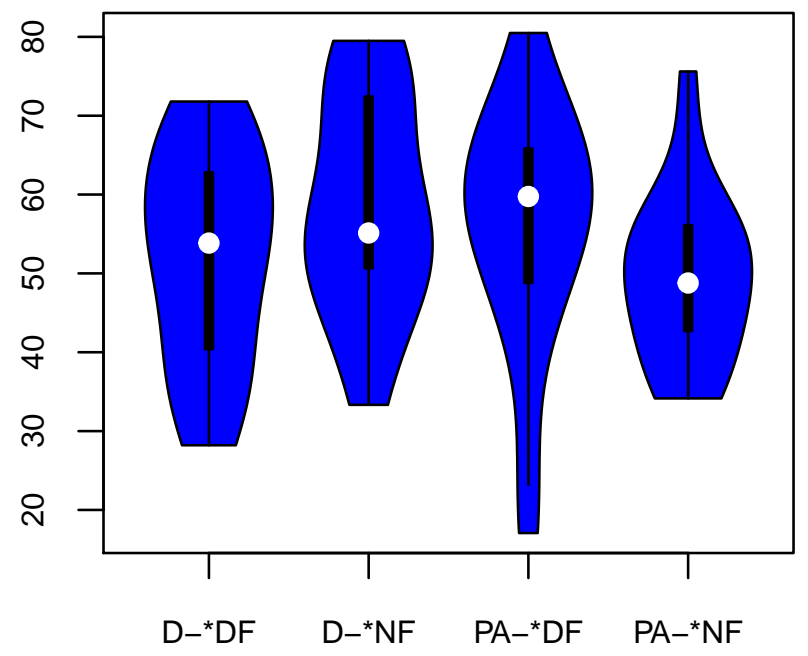

Rule Trained On - Constraint Tested On

Figure 6: Distribution of by-item violation preference for each item in Experiment 2.

the main effects of training and testing are not significant. Unlike in Experiment 1, the interaction of training and testing does not reach significance. The trend is in the predicted direction, but has not been confirmed statistically.

3.3 Discussion The results of Experiment 2 are inconclusive. The data show a trend in the same direction as Experiment 1, but the predicted interaction between training and testing is not significant. This could mean that the finding of Experiment 1 was indeed due to feedback, but it could also mean that without feedback, participants learned the generalization less well, resulting in noisier results. When variance increases, all else held equal, power decreases, so it is plausible that Experiment 2 failed to find a significant result due to a lack of power.

\section{General Discussion}

These experiments offer mixed support for the hypothesis that information about alternations is used in making phonotactic judgments. Experiment 1 found support for the hypothesis, but may have inadvertently drawn participants' attention to words that biased them towards giving such results. Experiment 2 eliminated feedback, and as a result the active rule was learned at a far lower rate. The criterion used to determine whether a participant had learned the rule sufficiently to be included in the analysis was lowered in an attempt to increase power, and this may have increased the noise in the results. The predicted interaction between training and testing was not significant, in contrast to Experiment 1. Further experimentation is needed to determine whether Experiment 2 merely lacked power or failed to show an effect because it the effect had been due to a confound.

Nevertheless, the trend found in Experiment 2 makes it worthwhile to consider the possibility that alternation-based knowledge affects phonotactics and spell out the implications if clearer evidence is found 
with further study. It would not show that alternations and phonotactics are necessarily computed from the same, monolithic system, as Optimality Theory asserts, but would argue that information is shared between them, at least from alternations to phonotactics. Although the findings of Pater \& Tessier (2003) are suggestive, this study cannot confirm the flow of information from phonotactics to alternations. However, of the two directions of information flow, that from alternations to phonotactics is the more surprising. Since phonotactic knowledge is likely to be acquired first, it may be helpful in acquiring alternations. The need for alternations to affect phonotactics is less obvious; phonologists may find it more plausible that the two forms of knowledge are in two-way communication than that alternations affects phonotactics but the reverse is not true.

\section{References}

Albright, Adam (2008). Gradient phonological acceptability as a grammatical effect. Http://web.mit.edu/albright/www/papers/Albright-GrammaticalGradience.pdf.

Albright, A. (2009). Feature-based generalisation as a source of gradient acceptability. Phonology 26:1, 9-41.

Bailey, T.M. \& U. Hahn (2001). Determinants of wordlikeness: Phonotactics or lexical neighborhoods? Journal of Memory and Language 44:4, 568-591.

Bates, Douglas \& Martin Maechler (2009). lme4: Linear mixed-effects models using S4 classes. URL http: / / CRAN . $\mathrm{R}$-project. org/package=lme4. R package version 0.999375-31.

Becker, M., A. Nevins \& N. Ketrez (2011). The surfeit of the stimulus: Analytic biases filter lexical statistics in Turkish laryngeal alternations. Language 87:1, 84-125.

Chomsky, Noam \& Morris Halle (1968). The Sound Pattern of English. Harper and Row, New York.

Coleman, J. \& J. Pierrehumbert (1997). Stochastic phonological grammars and acceptability. Computational Phonology: Third meeting of the ACL special interest group in computational phonology, 49-56.

Daland, R., B. Hayes, J. White, M. Garellek, A. Davis \& I. Norrmann (2011). Explaining sonority projection effects. Phonology 28:2, 197-234.

Darcy, Isabelle, Franck Ramus, Anne Christophe, Katherine Kinzler \& Emmanuel Dupoux (2009). Phonological knowledge in compensation for native and non-native assimilation. Variation and gradience in phonetics and phonology 14, p. 265, URL https://books.google.com/books?

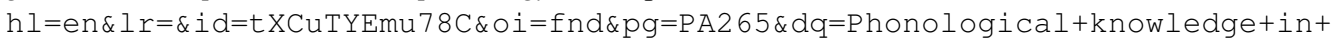
compen-+sation+for+native+and+non-native+assimilation . +\&ots=6rY7kCpEy $9 \&$ sig $=$ kCCnGKIYIURm0e-4pkkeMm4 9d3U.

Hayes, Bruce \& Colin Wilson (2008). A Maximum Entropy model of phonotactics and phonotactic learning. Linguistic Inquiry 39:3, 379-440.

Hayes, Bruce, Kie Zuraw, Péter Siptár \& Zsuzsa Cziráky Londe (2009). Natural and unnatural constraints in Hungarian. Language 85, 822-863.

Jakobson, Roman (1978). Mutual Assimilation of Russian Voiced and Voiceless Consonants. Studia Linguistica 32:1-2, 107-110, URL http://onlinelibrary.wiley.com/doi/10.1111/j.1467-9582.1978. tb00332.x/abstract.

Jarosz, Gaja (2006). Rich Lexicons and Restrictive Grammars - Maximum Likelihood Learning in Optimality Theory. Doctoral dissertation, Johns Hopkins University.

Jun, Jongho (2004). Place assimilation. Hayes, Bruce, Robert Kirchner \& Donca Steriade (eds.), Phonetically Based Phonology, Cambridge University Press, Cambridge, 58-86.

Kiparsky, Paul (1985). Some consequences of Lexical Phonology. Phonology 2, 85-138.

Kisseberth, Charles (1970). On the functional unity of phonological rules. Linguistic Inquiry 1, 291-306.

Pater, Joe \& Anne-Michelle Tessier (2003). Phonotactic knowledge and the acquisition of alternations. Solé, M. J., D. Recasens \& J. Romero (eds.), Proceedings of the 15th International Congress of Phonetic Sciences, Universitat Autònoma de Barcelona, Barcelona, 1177-1180.

Pierrehumbert, J. B (2006). The statistical basis of an unnatural alternation. Laboratory phonology 8 81-106.

Prince, Alan \& Paul Smolensky (1993/2004). Optimality Theory: Constraint Interaction in Generative Grammar. Malden, MA, and Oxford, UK: Blackwell.

R Development Core Team (2011). R: A Language and Environment for Statistical Computing. R Foundation for Statistical Computing, Vienna, Austria, URL http: / www.R-project.org/.

Tesar, Bruce \& Alan Prince (2004). Using phonotactics to learn phonological alternations. CLS 39, Part II: The Panels, Chicago Linguistic Society, Chicago.

Vitevitch, M.S. \& P.A. Luce (2004). A web-based interface to calculate phonotactic probability for words and nonwords in english. Behavior Research Methods 36:3, 481-487.

Wolf, Matthew (2008). Optimal interleaving: serial phonology-morphology interaction in a constraint-based model. Doctoral Dissertation, University of Massachusetts, Amherst, Amherst, Mass. 